\title{
Role for Leptin and Leptin Receptors in Stem Cells During Health and Diseases
}

\author{
Thao Trinh $^{1} \cdot$ Hal E. Broxmeyer ${ }^{1}$ (i) \\ Accepted: 31 January 2021 / Published online: 17 February 2021 \\ (C) The Author(s), under exclusive licence to Springer Science+Business Media, LLC part of Springer Nature 2021
}

\begin{abstract}
Hematopoietic stem cells (HSCs) give rise to all blood and immune cells in the body. These rare cells reside in the hypoxic niche of the bone marrow (BM) where they are subjected to a complex network of regulatory factors including cellular and molecular components. To sustain hematopoiesis over the lifetime of an individual, HSCs maintain distinctive metabolic programs, and in recent years nutritional factors have been increasingly recognized as critical regulators of HSC numbers and functions. Leptin (LEP), a neuroendocrine messenger, and its receptor (LEPR) are well-known for their immunomodulatory and energy balancing effects; yet, how LEP/LEPR signaling plays a role in hematopoiesis is under-appreciated. In this review, we summarize and highlight recent work that demonstrated involvement of LEP/LEPR in hematopoiesis under steady state or stress-associated situations as well as in pathological conditions such as cardiovascular diseases and malignancies. Although the field is only in its infancy, these studies suggest evidence of potential clinical applications and proof-of-principle for more in-depth future research.
\end{abstract}

Keywords Leptin $\cdot$ Leptin receptor $\cdot$ Hematopoietic stem cells $\cdot$ Hematopoiesis $\cdot$ Malignancy

\section{Introduction}

Discovered in the early 1990s as a key regulator of body weight and energy expenditure, leptin (LEP) has since been studied extensively in multidisciplinary fields [1-5]. Different organs are known producers of LEP including adipose tissue, placenta, stomach, etc. Hence, it is not surprising that LEP, a $16-\mathrm{kDa}$ polypeptide hormone, exerts both central and peripheral effects by binding to its surface receptor (LEPR) [6, 7]. LEPR exists in multiple isoforms as a result of alternative splicing; the longisoform $(O B-R b)$, but not the short isoforms $(O B-R a$, $O B-R c, O B-R d, O B-R e$ ), has been demonstrated to fully induce intracellular signaling mostly through the Janus kinase-signal transducer and activator of transcription (JAK/STAT) pathway $[8,9]$. As a neuroendocrine hormone, the effects of LEP on feeding behaviors are mediated in part through activation of proopiomelanocortin

Hal E. Broxmeyer

hbroxmey@iupui.edu

1 Departments of Microbiology/Immunology, Indiana University School of Medicine, 950 West Walnut Street, Bldg. R2, Room 302, Indianapolis, IN 46202-5121, USA neurons and inhibition of neuropeptide Y/agouti-related protein neurons in the hypothalamus [10-13]. However, its central effects are more intriguingly complex, and involve roles in depression, stress response, dopaminergic reward circuits, reproduction and thermoregulation. These topics have been reviewed in depth by others [14-19].

Being secreted mainly by adipocytes in proportion to body fat mass, LEP also acts as a proinflammatory adipokine. This is scientifically logical since immune functions are well-known to be tightly linked to nutritional status, and LEP is a critical messenger of the body energy storage throughout the entire life of an individual. It was reported early on that serum LEP levels and Lep mRNA expression increases following acute inflammation events or proinflammatory cytokine injection [20]. In fact, the roles of LEP and its signaling in mature immune cells have been well-investigated. For instance, starvation-induced immunosuppression and attenuated T-cell immunity were alleviated by treatment with LEP [21]. Consistent with this, human LEP acted as a negative regulator of regulatory $T$ (Treg) cells [22]. Diverse effects of LEP in different immune cells have been reviewed in previous publications [23-28]. For this review, we focus on roles of LEP/LEPR in hematopoiesis - under both homeostasis and malignant conditions - as well as in other stem cell systems. 


\section{LEP/LEPR in Hematopoiesis}

\section{LEP/LEPR in Adult Non-malignant Hematopoiesis, Via Microenvironmental Stromal Cells}

In the study of adult hematopoiesis under steady-state conditions, most work has focused on the roles of LEPR-expressing stromal cells in the bone marrow (BM) niches. This is not a great surprise since adipocytes, a potential major source of BM LEP, are themselves a critical component of niche cells [29-31]. It is wellappreciated that hematopoietic stem cells (HSCs) are maintained, nurtured and regulated by an intricate and highly dynamic network of both cellular and molecular factors [32-36]. In order to delineate specific cellular sources of important growth factors for HSC homeostasis, a stem cell factor $(S c f)$ green fluorescent protein $(G f p)$ knock-in murine model was employed to elegantly demonstrate that SCF from endothelial cells (ECs) and LEPRexpressing perivascular stromal cells were indispensable for BM HSC maintenance. In contrast, deletion of SCF from osteoblasts, Nestin-cre- or hematopoietic cells had insignificant effects on the BM HSC pool [37]. Notably, mesenchymal stem/stromal cells (MSCs) (including LEPR-expressing stromal cells) expressed SCF at higher levels than endothelial cells [38]. A follow-up study further demonstrated that SCF from endothelial cells was mainly critical for HSCs, whereas SCF from LEPRexpressing stromal cells was required not only by HSCs but also by a variety of progenitor cells including common lymphoid progenitors, common myeloid progenitors, megakaryocyte-erythrocyte progenitors and other more differentiated precursor cells [39].

To elucidate roles of niche cells, including $\mathrm{LEPR}^{+}$stromal cells, in context of hematopoietic recovery post-irradiation, a similar approach was utilized but to study the specific cellular source of pleiotrophin (PTN) rather than $\mathrm{SCF}$ in murine BM [40]. At baseline, using Ptn floxed/ floxed mice ( $P t n^{f l f l}$ mice) that were crossed with Cre-expressing mice to conditionally delete Ptn in different cell types, it was found that under homeostasis LEPR ${ }^{+}$stromal cells were an indispensable source of PTN required for HSC maintenance. In contrast, conditional deletion of Ptn from other cell types such as ECs, hematopoietic cells or osteoblasts did not affect HSC content nor HSC repopulating function in long-term competitive transplant assays. Interestingly, when mice were exposed to a sublethal dose of total body irradiation (500 cGy TBI), a shift in the roles of $\mathrm{LEPR}^{+}$stromal cells versus vascular endothelial cadherin (VE-cad) ${ }^{+}$ECs in hematopoietic recovery occurred. Even though Ptn deletion from either $\mathrm{LEPR}^{+}$ stromal cells or VE-cad ${ }^{+}$ECs resulted in reduction in numbers of $\mathrm{Lin}^{-} \mathrm{Sca}-{ }^{+} \mathrm{cKit}^{+}$(LSK; containing HSCs and progenitors) cells and myeloid progenitors by phenotyping analyses, only PTN from VE-cad ${ }^{+}$ECs was required for recovery of long-term (LT) -HSC. BM cells from irradiated VE-cad-Cre;Ptn ${ }^{f l / f l}$ but not LepR-Cre;Ptn ${ }^{f l f l}$ mice exhibited reduced repopulating potential in both primary and secondary transplants compared to irradiated control mice.

While these studies highly suggested that LEPR signaling was functionally critical for adult hematopoiesis particularly in the stromalniche, it would also be meaningful and clinically relevant to know whether the LEP ligand itself mediated these effects or if there were alternative cytokine cross-signaling pathways that acted through LEPR, for example like Interleukin (IL)-6 signaling [41, 42]. One reason being that obesity is a risk factor for hematologic malignancies. Patients with obesity have increased mortality rate and hematopoietic cell transplant (HCT)- associated complications. Moreover, altered LEP signaling has been well-documented in obesity [43]. In fact, the indirect involvement of LEP signaling on hematopoietic output by modulating BM stromal cells in the context of exercise and chronic inflammation such as in atherosclerosis and cardiovascular diseases has been reported [44]. Voluntary exercise induced HSC/hematopoietic progenitor cell (HPC) quiescent-promoting niche factors only in $\mathrm{LEPR}^{+}$stromal cells, but not in other niche cellular components whilereducing adipose-tissue-derivedLEP production. Also, LEP supplementation during exercise increased levels of circulating leukocytes, LSK cell proliferation and decreased gene expression of BM Angpt1, Cxcl12 and Vcam1, which are important for HSC/HPC quiescence and retention [44]. These findings were not mediated through LEP signaling onHSCs/HPCs; effects of exerciseremained unaffected in wild-type (WT) mice transplanted with $L e p R^{-/-}$BM from $d b$ / $d b$ mice. In contrast, cell-specific conditionaldeletion of $L e p R$ in stromal cells using $\operatorname{Pr} r x 1$-creER ${ }^{\mathrm{T} 2} ;$ Lepr $^{f l f f}$ mice showed a reduction in LSK cell proliferation and circulating leukocytes while enhancing expression of HSC maintenance niche factors [44]. This resembled the phenotype in exercising mice. Absence of LEP signaling in stromal cells of these mice alleviated the rise in LSK cell proliferation and systemic leukocytosis post-myocardial infarction (MI) [44]. Treatment with LEP-neutralizing antibody $30 \mathrm{~min}$ after MI reduced BM colony-forming granulocyte-macrophage progenitor cell proliferation and monocytes in the blood and at the site of the infarct. This resulted in less inflammation and fibrosis and helped accelerate recovery. Similar results were observed in adifferentmodel ofatherosclerosis [44]. Thesewell-designed studies highlighted the significant roles that LEPRexpressing stromal cells play in both steady-state and pathological hematopoiesis. Even though LEP/LEPR involvement in potential various pathological conditions in the context of hematopoiesis is only in its infancy, these findings may have therapeutic potential. 


\section{LEP/LEPR in Adult HSCs}

Not long after discoveries of LEP and its receptor, a novel sequence was cloned, namely B219, which was found in primitive hematopoietic cells isolated from the yolk sac, early fetal liver and some lymphohematopoietic cell lines. The sequence was expressed in at least four isoforms including one that resembled the recently published $L E P R$; its amino acid sequence shared similarity with granulocyte colony stimulating factor (G-CSF) receptor, gp130 and the leukemia inhibitory factor (LIF) receptor $[1,45]$. In that same year, cloning of different isoforms of LEPR from human hematopoietic cell cDNA libraries were reported [46]. Ectopic expression of the long isoform $O B-R b$ in murine $\mathrm{Ba} / \mathrm{F} 3$ and $\mathrm{M} 1$ cell lines respectively induced cell proliferation and differentiation. However, it was not clear whether LEP had effects on endogenously expressed LEPR and if so, in which hematopoietic cell populations. LEP enhanced cytokine production and phagocytosis by murine peritoneal macrophage [46]. While these findings were limited to in vitro work, they spurred interest in more recent years in the study of LEP and its receptor in the field of hematology.

Beyond the scope of LEPR in the BM microenvironment, the functional properties of LEPR-expressing HSCs were characterized in a recent publication (Fig. 1) [47]. Even though it's been known for years that a fraction of human $\mathrm{CD} 34^{+}$cells and mouse hematopoietic cells expressed LEPR, there was a gap in knowledge of how these cells might be functionally different from their LEPR ${ }^{-}$counterparts. $\mathrm{LEPR}^{+} \mathrm{LSK}$ cells, although occupying a much smaller percentage out of total LSK cells, were significantly enriched for both colony-forming progenitors and repopulating LTHSC as shown by higher engraftment and robust selfrenewing capacity in both primary and secondary competitive transplant assays [47]. Using a limiting dilution assay, LEPR $^{+}$SLAM HSCs (LSK CD $150^{+} \mathrm{CD} 48^{-}$) were compared to LEPR $^{-}$SLAM HSCs on a cell-to-cell basis. LEPR $^{+}$ SLAM HSCs consistently engrafted significantly higher than LEPR $^{-}$SLAM HSCs with a higher frequency of competitive repopulating units (CRUs, a measure of functional engrafting HSCs; on average, Poisson Statistical Analyses showed LEPR $^{+}$SLAM HSCs contained approximately 90 times more CRUs than LEPR ${ }^{-}$SLAM HSCs); multilineage differentiation capabilities were similar.

Consistent with previous findings that $\mathrm{CD} 45^{+}$hematopoietic cells hardly expressed the long isoform of $L e p R$ (or $O B$ $R b)$ [48], the majority of Lepr mRNA expressed by LEPR $^{+}$SLAM HSCs and LEPR ${ }^{+}$SLAM multipotent progenitor (MPP) (LSK CD150 ${ }^{-} \mathrm{CD}^{-} 8^{-}$) under steady-state were $O B-R a$ and $O B-R c$ in a bulk RNA-seq transcriptomic analysis. As noted in the study, the cells were sorted from freshly isolated BM from healthy young adult mice directly into lysis buffer for RNA extraction; hence, the isoforms of Lepr expressed in these cells might not exactly be the same as in those upon transplanted into a lethally irradiated host [47]. Whether LEPR ${ }^{+} H S C$ s express the long isoform (OB-Rb) at any stages during development or under other circumstances such as during stress hematopoiesis remains to be determined. Using a data-driven nonbiased approach, Fast Gene Set Enrichment Analysis (FGSEA) revealed the top hit pathways in LEPR ${ }^{+}$SLAM HSCs, compared to LEPR ${ }^{-}$SLAM HSCs, were Type-I Interferon and Interferon-gamma (IFN- $\gamma$ ) response pathways. In addition, among the top differential gene expression candidates were Relb and $c$-Rel; both are subunits of the nuclear factor kappa $b(\mathrm{NF}-\mathrm{kB})$ pathway. The IFN- $\gamma$ proinflammatory response pathway and NF- $\mathrm{KB}$ signaling have been implicated in development of embryonic HSCs $[49,50]$. Although these associations might suggest and explain a potential embryonic-like transcriptomic signature and the highly engrafting functional characteristic of $\mathrm{LEPR}^{+} \mathrm{LT}$ HSCs, in-depth mechanistic studies are warranted to shed light into the developmental origin of this subset of HSCs. Perhaps, it will be therapeutically relevant to determine whether LEP signaling plays a role in the transition of hemogenic ECs into HSCs in terms of both numbers and functions since the maternal status of energy storage, which is reflected and regulated by LEP levels, might influence hematopoiesis in the embryo. On the other hand, LEP resistance has been suggested to occur during aging, and if LEPR signaling has any role(s) in hematopoiesis it will be interesting to determine how aging may differentially affect LEPR ${ }^{+} \mathrm{HSCs}$ vs. LEPR ${ }^{-}$HSCs phenotypically and functionally in terms of engraftment and lineage choices [51, 52].

To the best of our knowledge, there has not been any evidences demonstrating direct effects of LEP/LEPR on HSCs or more immature progenitors in the literature. However, as mentioned earlier the roles of LEP/LEPR signaling in mature immune cells are well-established [24, 27], and because immune cells constitute a distinguishable hematopoietic cell niche [53, $54]$ it is conceivable that LEP could potentially regulate HSC/ HPC numbers and functions indirectly through the immune system especially in the context of stress hematopoiesis such as post-irradiation or in acute infections.

LEP exerts diverse effects on both innate and adaptive immune cells, and in general it is considered a proinflammatory cytokine with certain exceptions including its inhibitory effects on natural killer cells upon extensive exposure [55]. Within the adaptive immune branch, LEP is critical for $\mathrm{CD} 4^{+}$ $\mathrm{T}$ cell homeostasis and activation [56]. Interestingly, studies suggested that $\mathrm{CD}^{+} \mathrm{T}$ cells are important for hematopoiesis as well as successful engraftment of allogeneic BM [57, 58]. This poses a potential question that is whether LEP by activating $\mathrm{CD}^{+} \mathrm{T}$ cell can facilitate $\mathrm{HSC}$ reconstitution in the context of HCT. On the other hand, LEP negatively regulates Treg cells [59], but Treg cells were shown to play a crucial 
a Steady state

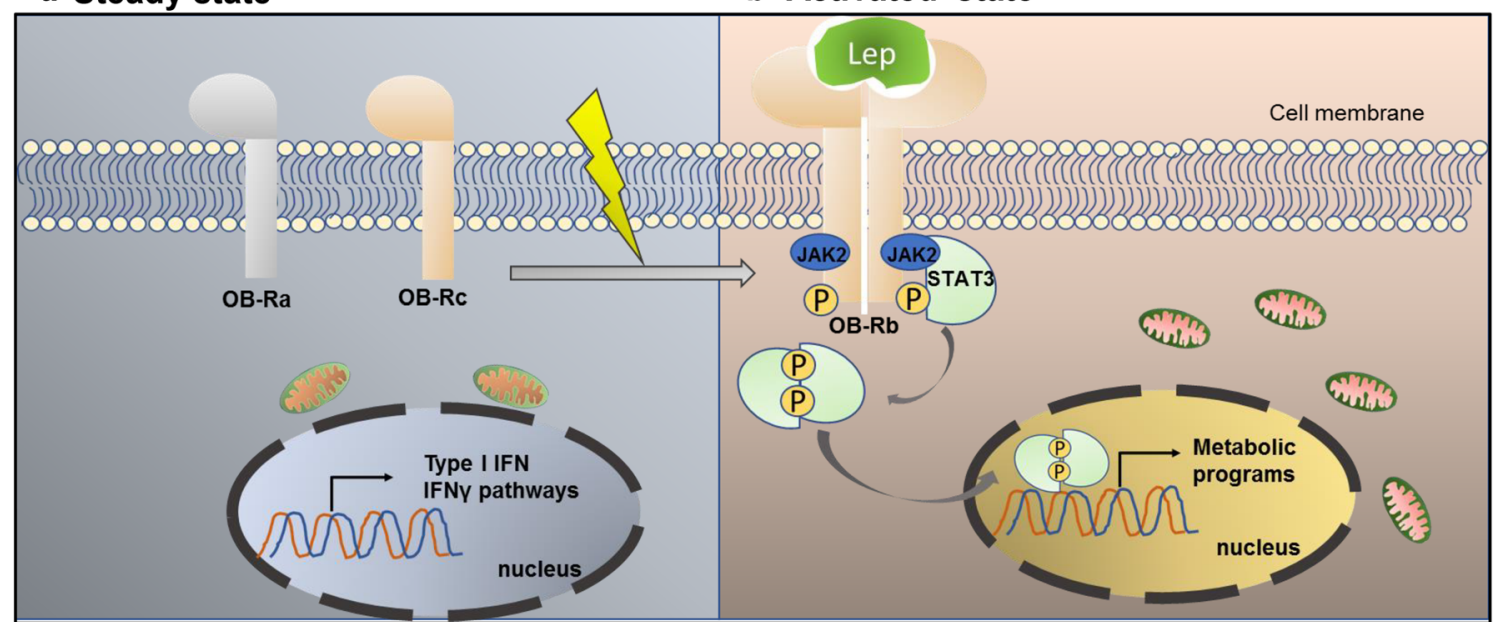

Fig. $1 \mathrm{LEPR}^{+} \mathrm{HSCs}$ under steady state condition [47] becomes 'activated' in post-injury recovery (hypothetical). a Under homeostasis $\mathrm{LEPR}^{+} \mathrm{HSC}$ only express short isoforms of Lepr $(O B-R a \& O B-R c)$ and are characterized by a proinflammatory transcriptomic profile. b Hypothetically, LEPR ${ }^{+}$HSCs may express long isoform of $\mathrm{Lepr}(\mathrm{OB}-$ $R b)$ as a result of stress-associated activation role in maintaining HSC quiescence [60]. Hence, it is possible that prolonged LEP stimulation could actually be detrimental to HSCs, and this could explain why obese patients, who are typically presented with hyperleptinemia, have a worse prognosis after allogeneic BM HCT [61]. Perhaps, the timing and dosing of LEP stimulation during a transplantation are what determines the outcomes - while the proinflammatory effects of LEP on immune cells could be important to the enhanced engraftment of HSCs, yet too much of it would be counterproductive.

During acute infections, LEP levels increased [62], and LEP has been well-characterized to activate innate immune cells as the first line of defense. Its effects include both direct and indirect involvements such as enhancement of phagocytosis by macrophages [63], stimulating secretion of proinflammatory cytokines including tumor necrosis factor alpha (TNF $\alpha$ ), IL-1 $\beta$ and IL-6 by monocytes [64], promoting neutrophil infiltration through chemotactic migration, and so on. Of note, innate myeloid cells are generally short-lived, so continuous increased replenishment from the BM through differentiation of myeloid precursors is needed. It is possible that LEP-mediated production of proinflammatory cytokines in response to an infection is amongst several mechanisms that create a feedback loop sustaining the so-called 'emergency myeloipoiesis.' Unfortunately, if this process keeps going excessively and/or is deregulated like what happens in cytokinerelease syndrome (CRS), such immunological responses become pathological and can cause severe or life-threatening sequelae [65]. The extent to which LEP is involved in this immunopathological condition can be potentially a question of great interest particularly in the ongoing pandemic of coronavirus disease 2019 (COVID-19) caused by the severe acute respiratory syndrome coronavirus 2 (SARS-CoV-2), as being overweight or obese has been consistently associated with more severe cases of COVID-19 [66-69]. In fact, chronically elevated levels of LEP in obesity and its consequential enhancement of inflammation both locally at the site of infection and systemically have been suggested to be at least partially responsible for this clinical observation [70-72]. It can be logically speculated that the enhanced proinflammatory effects of LEP at baseline in obesity helps exacerbates the virus-mediated cytokine storm. Interestingly, angiotensinconverting enzyme 2, the only so far known receptor for SARS-CoV-2, has been recently reported to be expressed by human cord blood-derived HSCs [73, 74]. Exposure to SARS-CoV-2 spike protein can activate the Nlrp3 inflammasome, which has been proposed to initiate CRS $[73,75]$, as well as negatively regulate HSC/HPC functional properties in vitro [74]. This is very important because the long-term effects of SARS-CoV-2 is still a subject of ongoing investigations, and it could potentially affect not only the infected individuals but also recipients of cellular therapies [76, 77]. To further understand how SARS-CoV-2 impacts hematopoiesis in individuals with aberrant LEP signaling including obese patients, future research is warranted because the effects of LEP could be multi-layered through its broad involvement in both immunity as well as metabolism.

\section{LEP/LEPR in Hematologic Malignancies - the Known and Unknown}

There have been numerous studies in patients with both myeloid and lymphoid malignancies that looked at changes in plasma LEP levels during the course of the diseases and showed leukemic cells expressing leptin receptor isoforms [78-87]. Unfortunately, the majority of these publications did not determine whether there were any distinct functional properties of LEPR-expressing leukemic cells or if LEP 
signaling played a role in human leukemia pathogenesis and maintenance, and if so, how. In this section, we highlight some of the work which provided proof-of-principle evidence of LEP and its receptor being possibly involved to some extent in different types of leukemias using: patient-derived samples, murine models and xenograft studies.

As early as 1999 , using reverse-transcriptase polymerase chain reaction to study gene expression levels, it was demonstrated that approximately $50 \%$ of collected patient specimens of either newly diagnosed primary or secondary acute myeloid leukemia (AML) expressed higher levels of the long isoform, than the short isoform, of $L E P R$. This was also true for primary and recurrent acute promyelocytic leukemia. In contrast, only the short isoform could be found in healthy human promyelocytes (defined as $\mathrm{CD} 34^{-} \mathrm{CD} 33^{+}$or $\mathrm{CD} 34^{-} \mathrm{CD} 13^{+}$). $L E P R$ was not detected in chronic or acute lymphocytic leukemic cells [78]. Patient-derived myeloid leukemic blasts and leukemic cell lines proliferated at low levels when treated with human recombinant LEP alone and significantly at higher levels in the presence of IL-3, G-CSF, or SCF [78].

Similarly, another paper characterized in vitro effects of LEP on cultured human AML blasts and compared systemic LEP levels in AML patients with healthy controls [88]. Serum LEP concentrations were significantly lower in untreated AML patients, but were not significantly different for patients in remission compared to normal subjects. Whether or not this change in serum LEP levels might have been related to the treatments was not further investigated. The authors [88] then looked at in vitro responses of patient-derived AML blasts (mixed CD34 $4^{+}$and $\mathrm{CD} 34^{-}$leukemic cells) to LEP treatment and found that LEP significantly increased blast cell proliferation and secretion of proinflammatory cytokines including IL-1 $\beta$, IL-6, TNF $\alpha$ and granulocyte-macrophage colonystimulating factor (GM-CSF) in a subset of patients. Notably, effects of LEP were observed at supraphysiological levels $(2 \mathrm{ug} / \mathrm{mL})$, so it is still unclear how these blasts might have behaved differently in response to physiological levels of leptin in vivo. Plus, systemic LEP concentrations may significantly differ from local sources such as in the leukemic BM niche. This was followed up with colony-forming unit assays using the same LEP concentration of $2 \mathrm{ug} / \mathrm{mL}$ in two different settings. Specifically, AML blasts were cultured for 7 days with or without LEP before being washed and plated, or the cells were plated directly in the presence or absence of LEP with GM-CSF and Erythropoietin. LEP was found to have minimal effects on colony formation, blast differentiation and apoptotic activity. However, results varied among patient samples. Overall, while these two studies were of some interest and suggested a potential new therapeutic target involving LEPR signaling in AML, the scope was limited to in vitro work, and the sizes of patient samples were relatively small. Thus, more in-depth future work with rigorous in vivo functional assessments and mechanistic insights are warranted.
In contrast to the above studies, more recent work [89] demonstrated that LEPR signaling mediated the protective effects of fasting on acute lymphoblastic leukemia (ALL), but not AML, cells. The study provided therapeutically relevant mechanisms. Using the activated Notch1 T-ALL model, the N-Myc B-ALL model and the MLL-AF9 AML model, it was found that fasting significantly suppressed ALL, but not AML, development by promoting blast cell differentiation and hence prolonged leukemia-induced mouse survival. RNA-seq analyses of leukemic B-ALL cells revealed expression levels of multiple cytokine receptors upregulated by fasting including Lepr and its major downstream effector, the transcription factor Stat3. This study [89] also pointed out that expression of $L E P R$ was positively correlated to survival rates in patients with pre-B-ALL. It was confirmed that B-ALL, T-ALL and AML leukemic cells express the long isoform $O B-R b$; likewise, fasting did not upregulate Lepr in AML as it did in B-ALL and T-ALL cells. To investigate potential role(s) that LEPR signaling plays in ALL development, WT mice were transplanted with N-Myc-infected BM $\mathrm{Lin}^{-}$cells that either came from WT $\left(\mathrm{Lepr}^{+/+}\right)$or $\mathrm{Lepr}^{-/}(\mathrm{db} /$ db) mice. Recipient mice that received Lepr $^{-/}$leukemiatransformed BM cells developed B-ALL at a significantly faster rate, had higher multi-organ leukemic burden and lower survival rate. The $\mathrm{Lepr}^{-/}$group had a higher percentage of $\mathrm{B} 220^{+} \mathrm{CD} 43^{+}$precursors and a lower percentage of differentiated $\mathrm{B} 220^{+} \mathrm{IgM}^{+}$cells, which suggested that LEPR signaling was critical for the differentiation of B-ALL cells. Similar results were observed in T-ALL but not in AML models [89]. Furthermore, the investigators repeated these experiments, but recipient mice were fasted for $48 \mathrm{~h}$ upon attaining about $60 \%$ of leukemic-transformed $\mathrm{GFP}^{+}$cells in PB. Consistent with initial findings, fasting attenuated percentages of $\mathrm{GFP}^{+}$leukemic cells and increased percentages of differentiated $\mathrm{IgM}^{+}$cells in the WT $\left(\mathrm{Lepr}^{+/+}\right)$, but not in $\mathrm{Lepr}^{-/}$, group. This confirmed that the anti-leukemic effects of fasting were mediated through LEPR signaling [89].

Mechanistically, LEPR overexpression in B-ALL cells led to upregulation of key transcription factors in B cell terminal differentiation including XBP1 and PRDM1 [89]. Overexpression of Prdml blocked B-ALL development, and knockdown of Prdm1 inhibited the anti-leukemic effect of LEPR overexpression in B-ALL. In silico analyses of patient databases showed that LEPR signaling and its related genes were positively correlated with better outcome and survival in pre-B-ALL leukemia. It was also demonstrated that fasting negatively regulated B-ALL development in a xenograft model transplanted with human pre-B-ALL cell line NALM-6, and it did so by upregulating LEPR expression [89].

A $\mathrm{Lep}^{-/}$mouse model $(\mathrm{Ob} / \mathrm{Ob})$ was used to investigate potential effects of obesity on development of AML from myeloid dysplastic syndrome (MDS) and its associated mortality rate [90]. Using BM from NUP98-HOXD13 transgenic 
mice, a model of MDS, compared to lean WT controls, obese $\mathrm{Lep}^{-1-}$ recipient mice had significantly higher survival rates, and opposite to the researchers' hypothesis these mice did not have increased risk of AML transformation. While $\mathrm{Lep}^{-/-}$ recipient mice transplanted with NUP98-HOXD13 BM had more severe monocytosis, it was suggested that their expanded adipose tissue was capable of harboring more myeloid cells, and hence reduced their detrimental accumulation in other vital organs such as the liver [90]. Even though the authors rationalized their reasons for excluding the potential effects of Lep knockout on their findings, it would have been of more interest if the phenotypes were consistent in a different model of obesity and NUP98-HOXD13 induced MDS. Furthermore, considering that obese individuals are at higher risk for leukemia development and obese children afflicted with leukemia have increased mortality rate [61,91-93], this study posed discrepancies between mice and humans, and thus requires future in-depth reevaluation.

In addition to being present on leukemic hematopoietic lineage cells, LEPR-expressing stromal cells have also been demonstrated to be involved in BM fibrosis of primary myelofibrosis (PMF), a subtype of myeloproliferative neoplasms [94]. While LEPR ${ }^{+}$mesenchymal stromal cells have been studied more extensively in normal hematopoiesis, the malignancy transformation potential of these prominent niche cells is far less understood. It was demonstrated that these cells were functionally and molecularly altered during the progression of thrombopoietin (TPO)-overexpressing-induced PMF [94]. Specifically, by using different genetic fate mapping models it was discovered that as the mice developed PMF, $\mathrm{LEPR}^{+}$stromal cells significantly expanded in numbers and increased production of collagen, which led to BM hematopoietic failure, fibrosis and extramedullary hematopoiesis. In line with this finding, gene expression analyses demonstrated that $\mathrm{LEPR}^{+}$stromal cells down-regulated HSC supporting growth factors including $C x c l 12$ and $S c f$ while up-regulating genes involved in fibrotic conversion. Importantly, mechanistic studies pinpointed platelet-derived growth factor receptor alpha (PDGFR $\alpha$ ) as the key player in the pathogenesis of bone marrow fibrosis caused by $\mathrm{LEPR}^{+}$stromal cells. This was therapeutically relevant since imatinib, an inhibitor of different tyrosine kinases including PDGFR $\alpha$, was shown to lessen fibrosis in this model of PMF [94]. Even though the study focused on PMF, it has opened up a new area for future studies into microenvironmental niche cells in other hematological malignancies, which is arguably just as important as studying the leukemic cells themselves.

\section{LEP/LEPR in Other Stem Cells}

The therapeutic value of LEP/LEPR in different stem cellbased systems have been explored over decades. Here, we describe some of the recent work that provided evidence of how LEP and its receptor regulate stem cell functions, with some showing potential clinical applications.

Thus far, most of the studies on LEP/LEPR in stem cell biology under homeostasis have not fully addressed mechanistically whether and how LEP/LEPR directly regulates stem cell functions. It was recently reported using mass spectrometry-based global proteomic analyses that LepR $R^{-/}$ (or $d b / d b$ ) mouse embryonic fibroblasts had increased levels of phosphorylated extracellular regulated MAP kinase (ERK) $1 / 2$, regulatory associated protein of mTOR (RAPTOR) and mitogen-activated protein kinase kinase (MEK) $1 / 2$ with the most differentially regulated pathways involving mitochondrial dysfunction and oxidative phosphorylation [95]. Likewise, induced pluripotent stem cells (iPSCs) derived from $d b / d b$ mice, and hence carrying the Lepr mutation, exhibited significant lowered protein levels of the pluripotency markers OCT4 and NANOG. $d b / d b$ iPSCs also revealed a proteomic profile that involved pathways regulating mitochondrial functions, protein synthesis (including upregulation of eukaryotic translation initiation factor $4 \mathrm{e}$ (EIF4E) and EIF4G) and pluripotency [95]. Molecularly, $d b /$ $d b$ iPSCs were demonstrated to have a reduction in STAT3 phosphorylation in response to LEP treatment even though at baseline there was no difference as compared to control iPSCs. Chromatin immunoprecipitation analysis revealed enrichment of Stat3 binding regions on the promoter of Eif4e of $d b / d b$ iPSCs, hence leading to enhanced protein synthesis as shown by a higher polysome/monosome ratio in polysome profiling study and an increased basal oxygen consumption rate in metabolic profiling study. Overall, these findings suggest a role(s) of LEP/LEPR both in embryonic stem cell development and in regulating pluripotency-associated genes. For future work, it will be interesting and relevant to investigate further functional characteristics of stem cells with disrupted LEP/LEPR signaling. For instance, how would it affect the ability of the cells to differentiate versus maintaining a stem-like state? Would it affect one or multiple lineages and to which extent? Would the effect(s) be reversible? Would the effect(s) be similar under steady state versus pathological conditions?

In a highly mechanistic study it was demonstrated that production of LEP induced by hypoxia preconditioning modulated the level of mitochondrial fusion protein OPA1, and hence improved human MSC survival and its therapeutic effects under ischemic conditions [96]. While the study showed that overexpression of LEP in human MSCs promoted their in vivo therapeutic effects including enhanced cardiac function and increased angiogenesis in cardiac infarction, it was unclear whether endogenous circulating LEP played any role, and if so, would endogenous LEP also act mainly through the GSK3/OMA1/OPA1 pathway? In a follow up study [97], it was shown that LEP increased glycolysis in MSCs 
by upregulating the glucose transporter sodium-glucose symporter 1 (SGLT1), and OPA1 was required for the effect of leptin on glycolysis. However, it was not well-addressed how OPA1 could be involved in the enhanced expression of SGLT1 [97]. Notably, LEP has been well-known for its angiogenic effect, and was shown to increase endothelial cell differentiation from murine embryonic stem cells [98, 99]. Furthermore, obese patients who have increased risk for cardiovascular diseases may be more likely to have higher systemic LEP levels and LEP resistance. Considering that this could also have a negative impact in vivo [44], it is important to weigh potential risks versus benefits as to how the cells may be preconditioned before transplantation.

On the other hand, LEP signaling has been studied extensively in context of solid tumor cancer stem cells (CSCs), particularly in breast cancer stem cells (BCSCs) $[100,101]$. CSCs represent one of the key challenges to cancer treatments; hence, understanding how CSCs are initiated and maintained by the tumor microenvironment will have an impact on development of more effective therapies. It has been demonstrated that LEPRb maintained stem-cell-like characteristics of triple negative breast cancer cells (TNBC) by inducing expression of stem cell self-renewal transcription factors OCT4, SOX2 and NANOG [102]. Using a NANOG promoter-driven GFP activity readout, it was observed that mouse LEPR when introduced into human TNBC MDAMB-231 and HCC70 non-CSCs induced NANOG expression [103]. Two other markers of CSCs SOX2 and OCT4 were also expressed at higher levels in LEPR-expressing nonCSCs as compared to control non-CSCs; this suggested that overexpression of LEPRb was sufficient to convert non-CSCs into CSCs. Still, it was not clear whether in situ non-CSCs can naturally acquire LEPRb expression to become CSCs and if the transition is reversible or not. While LEP signaling can have multiple downstream effectors, the authors showed that LEPRb specifically activated Tyr1077 and Tyr1138 phosphorylation, which in turn transactivated JAK2/STAT3, a major signaling pathway of LEPRb activation.

In a more mechanistic study, it was demonstrated that the pan-JAK inhibitor AZD1480 decreased viability of HCC1937 CSCs as compared to its non-CSC counterpart and suppressed tumorsphere formation from MCF7 CSCs [104]. To understand molecular pathways that governed this phenomenon, they performed RNAseq analyses on AZD1480-treated MCF7 tumorsphere cells and discovered that genes involved in lipid metabolism were downregulated [104]. Mechanistically, STAT3 modulated expression of genes involved in fatty acid oxidation (FAO) including the rate-limiting enzyme carnitinepalmitoyl transferase 1 (CPT1), which was required to maintain tumor cells in a stem-cell-like state. In line with this, inhibition of STAT3 in BCSCs reduced their self-renewing capacity. To further explore which upstream regulator affects STAT3/FAO pathway in BCSCs, they performed an adipokine array using breast adipocyte-conditioned medium. Among the most abundantly expressed adipokines was LEP. By using quantitative real time polymerase chain reaction and flow cytometry analyses, the only adipokine receptor detected at increased levels in the patient-derived BBM2 and BBM3 tumorsphere cells was LEPR. Consistent with this finding and the fact that LEPR signaling is known to activate JAK2/STAT3 pathway,

Table 1 LEP/LEPR in hematopoiesis. What is known?

\begin{tabular}{|c|c|}
\hline $\begin{array}{l}\text { LEP/LEPR in BM mesenchymal } \\
\text { stem/stromal cells (MSCs) }\end{array}$ & $\begin{array}{l}\text { - } \mathrm{LEPR}^{+} \text {BM MSCs were an indispensable source of SCF }(37,38) \text { and PTN }(40) \text { that were required to } \\
\text { maintain HSC homeostasis. } \\
\text { - SCF derived from LEPR }{ }^{+} \text {BM MSCs were critical for both HSCs and progenitor cells under steady state } \\
\text { ( } 39) \text {. } \\
\text { - Exercise induced expression of niche factors only in } \mathrm{LEPR}^{+} \text {BM MSCs while leptin supplementation } \\
\text { reversed this effect }(44) \text {. }\end{array}$ \\
\hline LEP/LEPR in HSC/HPCs & $\begin{array}{l}\text { - Isoforms of } L E P R \text { were found in both murine and human primitive hematopoietic cells }(45,47) \text {. } \\
\text { - LEP signaling through LEPR }{ }^{+} \text {BM MSCs induced LSK proliferation and hematopoietic output in } \\
\text { post-myocardial infarction and atherosclerosis (44). } \\
\text { - } \text { LEPR }^{+} \text {LSK cells were highly enriched for functional self-renewing LT-HSCs, and LEPR }{ }^{+} \text {HSCs repre- } \\
\text { sented a subset of more robustly repopulating LT-HSCs as compared to LEPR }{ }^{-} \text {HSCs }^{-}(47) \text {. }\end{array}$ \\
\hline LEP/LEPR in hematologic malignancies & $\begin{array}{l}\text { - A significant proportion of patient-derived leukemia samples expressed isoforms of } L E P R \text { (78-85). } \\
\text { - LEP induced myeloid leukemic blasts and cell lines to proliferate at low level while enhancing effects of } \\
\text { IL-3, G-CSF or SCF (78). } \\
\text { - At supraphysiological concentration, LEP increased patient-derived AML blast proliferation and secretion } \\
\text { of IL-6, TNF } \alpha \text {, IL-1 } \beta \text { and GM-CSF ( } 88) \text {. } \\
\text { - LEPR signaling mediated the anti-leukemic protective effects of fasting in B-ALL and T-ALL but not } \\
\text { AML models by promoting blast cell differentiation (89). } \\
\text { - LEPR }{ }^{+} \text {MSC alterations (e.g. lower expression of niche factors, upregulating genes involved in fibrosis) } \\
\text { played a key role in the pathogenesis of PMF (94). }\end{array}$ \\
\hline
\end{tabular}

Numbers in parenthesis ( ) refer to papers where this information can be found 
Table 2 LEP/LEPR in hematopoiesis. What's next?

Non-pathological conditions

Pathological conditions/leukemia o Molecular mechanisms by which LEPR signaling in stromal cells regulate expression of important niche factors under steady state vs. post-injury hematopoietic regeneration.

o Is LEPR on HSCs just a surface marker or is LEPR functional especially in context of post-injury recovery?

o Does the sources of LEP (e.g. systemic vs. local BM adipocytes) matter?

o Does LEP/LEPR play any roles in the specification of HSCs during embryonic period up until birth? And if so, by which mechanisms? And how does maternal and fetal leptin production affect these processes?

o How may aging exert differential effects on $\mathrm{LEPR}^{+}$stromal cells or LEPR ${ }^{+} \mathrm{HSCs}_{\mathrm{vs}} \mathrm{LEPR}^{-}$cells?

o Does LEP signal through other cytokine receptor(s) in the same family that may be present on BM MSCs and/or HSCs?

o Do $\mathrm{LEPR}^{+} \mathrm{HSCs} / \mathrm{HPCs}$ possess different potentials for leukemic transformation from $\mathrm{LEPR}^{-}$cells? How can other risk factors possibly further modulate the difference?

o There were discrepancies between MDS model using $\mathrm{Lep}^{-/-}$mice and patient studies. Was this actually due to obesity alone as suggested or the absence of LEP played additional roles?

o While healthy mouse HSCs/MPPs were reported to express predominantly only the short isoforms under steady state, by which mechanisms the leukemic blast cells for both myeloid and lymphoid malignancies reactivated LEPR long isoform? And how does this affect their functional behaviors? Implications for treatments? further experiments revealed that adding LEP to culture media increased tumorsphere formation, as well as phosphorylation of JAK2 and STAT3. Likewise, treatment with LEP neutralizing antibody reversed the increase in expression of $C P T 1 B$ and $A C A D M$, both enzymes involved in the FAO pathway, and reduced the percentage of BCSCs in the breast tumors. In fact, in patient tumor samples it was found that chemoresistant metastatic breast tumor sections expressed higher level of LEPR with LEPR-positive cells having increased CPT1B expression. Altogether, these in-depth mechanistic studies using patient-derived samples strongly suggested that leptin receptor and its downstream effectors JAK2/STAT3/CPT1 may be potential drug targets that will specifically eradicate the chemoresistant BCSCs.

\section{Concluding Remarks}

LEP and LEPR signaling play important modulatory roles throughout an organism's life from conception to old age. LEPR resistance is well-recognized in obesity and aging [43, 105]. With a persistently increased prevalence of obesity and growth in the elderly populations worldwide, understanding how the different pathological conditions including hematologic malignancies and hematopoietic transplantationassociated complications biasedly affected these subsets of patients has become even more important. In this report, we noted and highlighted the work that provided proof-ofprinciple evidence for more future in-depth studies. A summary of what is known about LEP and LEPR in hematopoiesis can be found in Table 1. In brief, LEP/LEPR have been suggested to regulate stem cell numbers and functions particularly in the hematopoietic system under homeostasis, as well as post-injury regeneration and malignant transformation. Even though there are still some discrepancies between different studies or between animal and human data especially regarding LEPR and leukemic cells, the findings lay a strong foundation and create interesting questions to be answered in follow-up studies (Table 2). Future work that elucidates and provides insight into mechanisms of LEP/LEPR interactions is warranted and may provide potential implications for therapeutic approaches to enhance healthcare.

Abbreviations ALL, acute lymphoblastic leukemia; AML, acute myeloid leukemia; BCSC, breast cancer stem cell; BM, bone marrow; COVID-19, coronavirus disease 2019; CPT1, carnitinepalmitoyl transferase 1 ; CRS, cytokine release syndrome; CRU, competitive repopulating unit; CSC, cancer stem cell; EC, endothelial cell; EIF, eukaryotic initiation factor; ERK, extracellular regulated MAP kinase; FAO, fatty acid oxidation; FGSEA, fast gene set enrichment analysis; G-CSF, granulocyte colony stimulating factor; GFP, green fluorescence protein; GMCSF, granulocyte-macrophage colony-stimulating factor; HCT, hematopoietic cell transplant; HPC, hematopoietic progenitor cell; HSC, hematopoietic stem cell; IFN, interferon; IL, interleukin; iPSC, induced pluripotent stem cell; JAK/STAT, Janus kinase-signal transducer and activator of transcription; LEP, leptin; LEPR, leptin receptor; LIF, leukemia inhibitory factor; LSK, Lineage(Lin) ${ }^{-} \mathrm{Scal}^{+}{ }^{+} \mathrm{CKit}^{+}$; LT, long-term; MDS, myeloid dysplastic syndrome; MEK, Mitogen-activated protein kinase kinase; MI, myocardial infarction; MPP, multipotent progenitor; MSC, mesenchymal stem cell; NF- $\mathrm{B}$, nuclear factor kappa $b$; PDGFR $\alpha$, plateletderived growth factor receptor alpha; PMF, primary myelofibrosis; PRDM1, PR domain containing 1; PTN, pleiotrophin; RAPTOR, regulatory associated protein of mTOR; SARS-CoV-2, severe acute respiratory syndrome coronavirus 2; SCF, stem cell factor; SGLT1, sodiumglucose symporter 1; TNBC, triple negative breast cancer cell; TNF, tumor necrosis factor; TPO, thrombopoietin; Treg, regulatory T; VEcad, vascular endothelial cadherin; WT, wild-type; XBP1, X-box binding protein 1

Author Contributions TT performed literature search and drafted the manuscript. HEB provided suggestions and revised the drafts.

Funding HEB was supported by US Public Health Service grants from the National Institutes of Health: R35 HL139599, R01 DK109188, T32 DK007519, and U54 DK106846. TT was supported by Training grant T32 DK007519. 
Data Availability Not applicable to this review.

\section{Declarations}

Ethical Approval Not applicable to this review.

Consent to Participate Not applicable to this review.

Consent to Publish Not applicable to this review.

Conflict of Interest None of the authors have conflicts of interest with this paper.

\section{References}

1. Bahary, N., Leibel, R. L., Joseph, L., \& Friedman, J. M. (1990). Molecular mapping of the mouse $\mathrm{db}$ mutation. Proceedings of the National Academy of Sciences of the United States of America, 87(21), 8642-8646. https://doi.org/10.1073/pnas.87.21.8642.

2. Friedman, J. M., Leibel, R. L., Siegel, D. S., Walsh, J., \& Bahary, N. (1991). Molecular mapping of the mouse ob mutation. Genomics, 11(4), 1054-1062. https://doi.org/10.1016/08887543(91)90032-a.

3. Zhang, Y., Proenca, R., Maffei, M., Barone, M., Leopold, L., \& Friedman, J. M. (1994). Positional cloning of the mouse obese gene and its human homologue. Nature, 372(6505), 425-432. https://doi.org/10.1038/372425a0.

4. Friedman, J. M., \& Halaas, J. L. (1998). Leptin and the regulation of body weight in mammals. Nature, 395(6704), 763-770. https:// doi.org/10.1038/27376.

5. Tartaglia, L. A., Dembski, M., Weng, X., Deng, N., Culpepper, J., Devos, R.,.. . Tepper, R. I. (1995). Identification and expression cloning of a leptin receptor, OB-R. Cell, 83(7), 1263-1271. https://doi.org/10.1016/0092-8674(95)90151-5.

6. Campfield, L. A., Smith, F. J., Guisez, Y., Devos, R., \& Burn, P. (1995). Recombinant mouse OB protein: evidence for a peripheral signal linking adiposity and central neural networks. Science, 269(5223), 546-549. https://doi.org/10.1126/science.7624778.

7. Zhou, Y., \& Rui, L. (2013). Leptin signaling and leptin resistance. Frontiers in Medicine, 7(2), 207-222. https://doi.org/10.1007/ s11684-013-0263-5.

8. Lee, G. H., Proenca, R., Montez, J. M., Carroll, K. M., Darvishzadeh, J. G., Lee, J. I., \& Friedman, J. M. (1996). Abnormal splicing of the leptin receptor in diabetic mice. Nature, 379(6566), 632-635. https://doi.org/10.1038/379632a0.

9. Gorska, E., Popko, K., Stelmaszczyk-Emmel, A., Ciepiela, O., Kucharska, A., \& Wasik, M. (2010). Leptin receptors. European Journal of Medical Research, 15(Suppl 2), 50-54. https://doi.org/ 10.1186/2047-783x-15-s2-50.

10. Smith, F. J., Campfield, L. A., Moschera, J. A., Bailon, P. S., \& Burn, P. (1998). Brain administration of OB protein (leptin) inhibits neuropeptide-Y-induced feeding in ob/ob mice. Regulatory Peptides, 75-76, 433-439. https://doi.org/10.1016/s01670115(98)00099-8.

11. Inui, A. (1999). Feeding and body-weight regulation by hypothalamic neuropeptides-mediation of the actions of leptin. Trends in Neurosciences, 22(2), 62-67. https://doi.org/10.1016/s01662236(98)01292-2.

12. Cheung, C. C., Clifton, D. K., \& Steiner, R. A. (1997). Proopiomelanocortin neurons are direct targets for leptin in the hypothalamus. Endocrinology, 138(10), 4489-4492. https://doi. org/10.1210/endo.138.10.5570.
13. Thornton, J. E., Cheung, C. C., Clifton, D. K., \& Steiner, R. A. (1997). Regulation of hypothalamic proopiomelanocortin mRNA by leptin in ob/ob mice. Endocrinology, 138(11), 5063-5066. https://doi.org/10.1210/endo.138.11.5651.

14. Friedman, J. (2016). The long road to leptin. The Journal of Clinical Investigation, 126(12), 4727-4734. https://doi.org/10. 1172/JCI91578.

15. Chou, S. H., \& Mantzoros, C. (2014). 20 years of leptin: role of leptin in human reproductive disorders. The Journal of Endocrinology, 223(1), T49-T62. https://doi.org/10.1530/JOE14-0245.

16. Barb, C. R., Hausman, G. J., \& Lents, C. A. (2008). Energy metabolism and leptin: effects on neuroendocrine regulation of reproduction in the gilt and sow. Reproduction in Domestic Animals, 43(Suppl 2), 324-330. https://doi.org/10.1111/j.1439-0531.2008. 01173.x.

17. Schneider, J. E. (2004). Energy balance and reproduction. Physiology \& Behavior, 81(2), 289-317. https://doi.org/10. 1016/j.physbeh.2004.02.007.

18. Friedman, J. M. (2019). Leptin and the endocrine control of energy balance. Nature Metabolism, 1(8), 754-764. https://doi.org/10. 1038/s42255-019-0095-y.

19. Ramos-Lobo, A. M., \& Donato, J. Jr. (2017). The role of leptin in health and disease. Temperature (Austin), 4(3), 258-291. https:// doi.org/10.1080/23328940.2017.1327003.

20. Sarraf, P., Frederich, R. C., Turner, E. M., Ma, G., Jaskowiak, N. T., Rivet, D. J. III, \& Alexander, H. R. (1997). Multiple cytokines and acute inflammation raise mouse leptin levels: potential role in inflammatory anorexia. The Journal of Experimental Medicine, 185(1), 171-175. https://doi.org/10.1084/jem.185.1.171.

21. Lord, G. M., Matarese, G., Howard, J. K., Baker, R. J., Bloom, S. R., \& Lechler, R. I. (1998). Leptin modulates the T-cell immune response and reverses starvation-induced immunosuppression. Nature, 394(6696), 897-901. https://doi.org/10.1038/29795.

22. De Rosa, V., Procaccini, C., Cali, G., Pirozzi, G., Fontana, S., Zappacosta, S.,... . Matarese, G. (2007). A key role of leptin in the control of regulatory $\mathrm{T}$ cell proliferation. Immunity, 26(2), 241-255. https://doi.org/10.1016/j.immuni.2007.01.011.

23. Gruver, A. L., \& Sempowski, G. D. (2008). Cytokines, leptin, and stress-induced thymic atrophy. Journal of Leukocyte Biology, 84(4), 915-923. https://doi.org/10.1189/jlb.0108025.

24. Naylor, C., \& Petri, W. A., Jr. (2016). Leptin regulation of immune responses. Trends in Molecular Medicine, 22(2), 88-98. https://doi.org/10.1016/j.molmed.2015.12.001.

25. Perez-Perez, A., Vilarino-Garcia, T., Fernandez-Riejos, P., Martin-Gonzalez, J., Segura-Egea, J. J., \& Sanchez-Margalet, V. (2017). Role of leptin as a link between metabolism and the immune system. Cytokine \& Growth Factor Reviews, 35, 71-84. https://doi.org/10.1016/j.cytogfr.2017.03.001.

26. Abella, V., Scotece, M., Conde, J., Pino, J., Gonzalez-Gay, M. A., Gomez-Reino, J. J., \& Gualillo, O. (2017). Leptin in the interplay of inflammation, metabolism and immune system disorders. Nature Reviews Rheumatology, 13(2), 100-109. https://doi.org/ 10.1038/nrrheum.2016.209.

27. Francisco, V., Pino, J., Campos-Cabaleiro, V., Ruiz-Fernandez, C., Mera, A., Gonzalez-Gay, M. A., \& Gualillo, O. (2018). Obesity, fat mass and immune system: role for leptin. Frontiers in Physiology, 9, 640. https://doi.org/10.3389/fphys.2018.00640.

28. La Cava, A., \& Matarese, G. (2004). The weight of leptin in immunity. Nature Reviews. Immunology, 4(5), 371-379. https:// doi.org/10.1038/nri1350.

29. Laharrague, P., Larrouy, D., Fontanilles, A. M., Truel, N., Campfield, A., Tenenbaum, R., \& Casteilla, L. (1998). High expression of leptin by human bone marrow adipocytes in primary culture. FASEB Journal, 12(9), 747-752. https://doi.org/10.1096/ fasebj.12.9.747. 
30. Zhou, B. O., Yu, H., Yue, R., Zhao, Z., Rios, J. J., Naveiras, O., \& Morrison, S. J. (2017). Bone marrow adipocytes promote the regeneration of stem cells and haematopoiesis by secreting SCF. Nature Cell Biology, 19(8), 891-903. https://doi.org/10.1038/ ncb3570.

31. Naveiras, O., Nardi, V., Wenzel, P. L., Hauschka, P. V., Fahey, F., \& Daley, G. Q. (2009). Bone-marrow adipocytes as negative regulators of the haematopoietic microenvironment. Nature, 460(7252), 259-263. https://doi.org/10.1038/nature08099.

32. Broxmeyer, H. E., Hoggatt, J., O'Leary, H. A., Mantel, C., Chitteti, B. R., Cooper, S., \& Campbell, T. B. (2012). Dipeptidylpeptidase 4 negatively regulates colony-stimulating factor activity and stress hematopoiesis. Nature Medicine, 18(12), 1786-1796. https://doi.org/10.1038/nm.2991.

33. Sarkaria, S. M., Decker, M., \& Ding, L. (2018). Bone marrow micro-environment in normal and deranged hematopoiesis: opportunities for regenerative medicine and therapies. Bioessays, 40(3). https://doi.org/10.1002/bies.201700190.

34. Broxmeyer, H. E., Li, J., Hangoc, G., Cooper, S., Tao, W., Mantel, C., \& de Sauvage, F. J. (2007). Regulation of myeloid progenitor cell proliferation/survival by IL-31 receptor and IL-31. Experimental Hematology, 35(4 Suppl 1), 78-86. https://doi.org/ 10.1016/j.exphem.2007.01.028.

35. Scadden, D. T. (2014). Nice neighborhood: emerging concepts of the stem cell niche. Cell, 157(1), 41-50. https://doi.org/10.1016/j. cell.2014.02.013.

36. Morrison, S. J., \& Scadden, D. T. (2014). The bone marrow niche for haematopoietic stem cells. Nature, 505(7483), 327-334. https://doi.org/10.1038/nature12984.

37. Ding, L., Saunders, T. L., Enikolopov, G., \& Morrison, S. J. (2012). Endothelial and perivascular cells maintain haematopoietic stem cells. Nature, 481(7382), 457-462. https:// doi.org/10.1038/nature10783.

38. Asada, N., Kunisaki, Y., Pierce, H., Wang, Z., Fernandez, N. F., Birbrair, A., \& Frenette, P. S. (2017). Differential cytokine contributions of perivascular haematopoietic stem cell niches. Nature Cell Biology, 19(3), 214-223. https://doi.org/10.1038/ncb3475.

39. Comazzetto, S., Murphy, M. M., Berto, S., Jeffery, E., Zhao, Z., \& Morrison, S. J. (2019). Restricted hematopoietic progenitors and erythropoiesis require SCF from leptin receptor + niche cells in the bone marrow. Cell Stem Cell, 24(3), 477-486 e476. https://doi.org/ 10.1016/j.stem.2018.11.022.

40. Himburg, H. A., Termini, C. M., Schlussel, L., Kan, J., Li, M., Zhao, L., \& Chute, J. P. (2018). Distinct bone marrow sources of pleiotrophin control hematopoietic stem cell maintenance and regeneration. Cell Stem Cell, 23(3), 370-381 e375. https://doi.org/ 10.1016/j.stem.2018.07.003.

41. Sadagurski, M., Norquay, L., Farhang, J., D’Aquino, K., Copps, K., \& White, M. F. (2010). Human IL6 enhances leptin action in mice. Diabetologia, 53(3), 525-535. https://doi.org/10.1007/ s00125-009-1580-8.

42. Baumann, H., Morella, K. K., White, D. W., Dembski, M., Bailon, P. S., Kim, H., \& Tartaglia, L. A. (1996). The full-length leptin receptor has signaling capabilities of interleukin 6-type cytokine receptors. Proceedings of the National Academy of Sciences of the United States of America, 93(16), 8374-8378. https://doi.org/10. 1073/pnas.93.16.8374.

43. Myers, M. G., Jr., Leibel, R. L., Seeley, R. J., \& Schwartz, M. W. (2010). Obesity and leptin resistance: distinguishing cause from effect. Trends in Endocrinology and Metabolism, 21(11), 643651. https://doi.org/10.1016/j.tem.2010.08.002.

44. Frodermann, V., Rohde, D., Courties, G., Severe, N., Schloss, M. J., Amatullah, H., \& Nahrendorf, M. (2019). Exercise reduces inflammatory cell production and cardiovascular inflammation via instruction of hematopoietic progenitor cells. Nature
Medicine, 25(11), 1761-1771. https://doi.org/10.1038/s41591019-0633-x.

45. Cioffi, J. A., Shafer, A. W., Zupancic, T. J., Smith-Gbur, J., Mikhail, A., Platika, D., \& Snodgrass, H. R. (1996). Novel B219/OB receptor isoforms: possible role of leptin in hematopoiesis and reproduction. Nature Medicine, 2(5), 585-589. https:// doi.org/10.1038/nm0596-585.

46. Gainsford, T., Willson, T. A., Metcalf, D., Handman, E., McFarlane, C., Ng, A., \& Hilton, D. J. (1996). Leptin can induce proliferation, differentiation, and functional activation of hemopoietic cells. Proceedings of the National Academy of Sciences of the United States of America, 93(25), 14564-14568. https://doi. org/10.1073/pnas.93.25.14564.

47. Trinh, T., Ropa, J., Aljoufi, A., Cooper, S., Sinn, A., Srour, E. F., Broxmeyer, H. E. (2020). Leptin receptor, a surface marker for a subset of highly engrafting long-term functional hematopoietic stem cells. Leukemia. https://doi.org/10.1038/s41375-02001079-z.

48. Zhou, B. O., Yue, R., Murphy, M. M., Peyer, J. G., \& Morrison, S. J. (2014). Leptin-receptor-expressing mesenchymal stromal cells represent the main source of bone formed by adult bone marrow. Cell Stem Cell, 15(2), 154-168. https://doi.org/10.1016/j.stem. 2014.06.008.

49. Sawamiphak, S., Kontarakis, Z., \& Stainier, D. Y. (2014). Interferon gamma signaling positively regulates hematopoietic stem cell emergence. Developmental Cell, 31(5), 640-653. https://doi.org/10.1016/j.devcel.2014.11.007.

50. Espin-Palazon, R., \& Traver, D. (2016). The NF-kappaB family: Key players during embryonic development and HSC emergence. Experimental Hematology, 44(7), 519-527. https://doi.org/10. 1016/j.exphem.2016.03.010.

51. Broxmeyer, H. E., Liu, Y., Kapur, R., Orschell, C. M., Aljoufi, A., Ropa, J. P., \& Capitano, M. L. (2020). Fate of hematopoiesis during aging. What do we really know, and what are its implications? Stem Cell Reviews and Reports, 16(6), 1020-1048. https:// doi.org/10.1007/s12015-020-10065-y.

52. Gabriely, I., Ma, X. H., Yang, X. M., Rossetti, L., \& Barzilai, N. (2002). Leptin resistance during aging is independent of fat mass. Diabetes, 51(4), 1016-1021. https://doi.org/10.2337/diabetes.51. 4.1016.

53. Riether, C., Schurch, C. M., \& Ochsenbein, A. F. (2015). Regulation of hematopoietic and leukemic stem cells by the immune system. Cell Death and Differentiation, 22(2), 187-198. https://doi.org/10.1038/cdd.2014.89.

54. Wei, Q., \& Frenette, P. S. (2018). Niches for hematopoietic stem cells and their progeny. Immunity, 48(4), 632-648. https://doi.org/ 10.1016/j.immuni.2018.03.024.

55. Wrann, C. D., Laue, T., Hubner, L., Kuhlmann, S., Jacobs, R., Goudeva, L., \& Nave, H. (2012). Short-term and long-term leptin exposure differentially affect human natural killer cell immune functions. American Journal of Physiology. Endocrinology and Metabolism, 302(1), E108-E116. https://doi.org/10.1152/ ajpendo.00057.2011.

56. Saucillo, D. C., Gerriets, V. A., Sheng, J., Rathmell, J. C., \& Maciver, N. J. (2014). Leptin metabolically licenses T cells for activation to link nutrition and immunity. Journal of Immunology, 192(1), 136-144. https://doi.org/10.4049/jimmunol.1301158.

57. Monteiro, J. P., Benjamin, A., Costa, E. S., Barcinski, M. A., \& Bonomo, A. (2005). Normal hematopoiesis is maintained by activated bone marrow CD4 + T cells. Blood, 105(4), 1484-1491. https://doi.org/10.1182/blood-2004-07-2856.

58. Kaufman, C. L., Colson, Y. L., Wren, S. M., Watkins, S., Simmons, R. L., \& Ildstad, S. T. (1994). Phenotypic characterization of a novel bone marrow-derived cell that facilitates engraftment of allogeneic bone marrow stem cells. Blood, 84(8), 24362446. https://doi.org/10.4161/onci.26586. 
59. Zeng, H., \& Chi, H. (2013). The interplay between regulatory T cells and metabolism in immune regulation. Oncoimmunology, 2(11), e26586. https://doi.org/10.4161/onci.26586.

60. Hirata, Y., Furuhashi, K., Ishii, H., Li, H. W., Pinho, S., Ding, L., \& Fujisaki, J. (2018). CD150(high) bone marrow tregs maintain hematopoietic stem cell quiescence and immune privilege via adenosine. Cell Stem Cell, 22(3), 445-453 e445. https://doi.org/ 10.1016/j.stem.2018.01.017.

61. Weiss, B. M., Vogl, D. T., Berger, N. A., Stadtmauer, E. A., \& Lazarus, H. M. (2013). Trimming the fat: obesity and hematopoietic cell transplantation. Bone Marrow Transplantation, 48(9), 1152-1160. https://doi.org/10.1038/bmt.2012.201.

62. Behnes, M., Brueckmann, M., Lang, S., Putensen, C., Saur, J., Borggrefe, M., \& Hoffmann, U. (2012). Alterations of leptin in the course of inflammation and severe sepsis. BMC Infectious Diseases, 12, 217. https://doi.org/10.1186/1471-2334-12-217.

63. Mancuso, P., Myers, M. G., Jr., Goel, D., Serezani, C. H., O'Brien, E., Goldberg, J., \& Peters-Golden, M. (2012). Ablation of leptin receptor-mediated ERK activation impairs host defense against Gram-negative pneumonia. Journal of Immunology, 189(2), 867-875. https://doi.org/10.4049/jimmunol.1200465.

64. Tsiotra, P. C., Boutati, E., Dimitriadis, G., \& Raptis, S. A. (2013). High insulin and leptin increase resistin and inflammatory cytokine production from human mononuclear cells. BioMed Research International, 2013, 487081. https://doi.org/10.1155/ 2013/487081.

65. Moore, J. B., \& June, C. H. (2020). Cytokine release syndrome in severe COVID-19. Science, 368(6490), 473-474. https://doi.org/ 10.1126/science.abb8925.

66. Kass, D. A., Duggal, P., \& Cingolani, O. (2020). Obesity could shift severe COVID-19 disease to younger ages. Lancet, 395(10236), 1544-1545. https://doi.org/10.1016/S01406736(20)31024-2.

67. Simonnet, A., Chetboun, M., Poissy, J., Raverdy, V., Noulette, J., Duhamel, A.,.. . Obesity study, g. (2020). High prevalence of obesity in severe acute respiratory syndrome coronavirus-2 (SARS-CoV-2) requiring invasive mechanical ventilation. Obesity (Silver Spring), 28(7), 1195-1199. https://doi.org/10. 1002/oby.22831.

68. Sacco, V., Rauch, B., Gar, C., Haschka, S., Potzel, A. L., KernMatschilles, S.,.. . Lechner, A. (2020). Overweight/obesity as the potentially most important lifestyle factor associated with signs of pneumonia in COVID-19. PLoS One, 15(11), e0237799. https:// doi.org/10.1371/journal.pone.0237799.

69. Lockhart, S. M., \& O'Rahilly, S. (2020). When two pandemics meet: why is obesity associated with increased COVID-19 mortality? Med (New York), 1(1), 33-42. https://doi.org/10.1016/j. medj.2020.06.005.

70. Rebello, C. J., Kirwan, J. P., \& Greenway, F. L. (2020). Obesity, the most common comorbidity in SARS-CoV-2: is leptin the link? International Journal of Obesity, 44(9), 1810-1817. https://oi. org/10.1038/s41366-020-0640-5.

71. van der Voort, P. H. J., Moser, J., Zandstra, D. F., Muller Kobold, A. C., Knoester, M., Calkhoven, C. F.,.. . van Meurs, M. (2020). Leptin levels in SARS-CoV-2 infection related respiratory failure: A cross-sectional study and a pathophysiological framework on the role of fat tissue. Heliyon, 6(8), e04696. https://doi.org/10. 1016/j.heliyon.2020.e04696.

72. Wang, J., Xu, Y., Zhang, X., Wang, S., Peng, Z., Guo, J., \& Liu, Z. (2021). Leptin correlates with monocytes activation and severe condition in COVID-19 patients. Journal of Leukocyte Biology. https://doi.org/10.1002/JLB.5HI1020-704R.

73. Ratajczak, M. Z., Bujko, K., Ciechanowicz, A., Sielatycka, K., Cymer, M., Marlicz, W., \& Kucia, M. (2020). SARS-CoV-2 entry receptor ACE2 is expressed on very small CD45(-) precursors of hematopoietic and endothelial cells and in response to virus spike protein activates the Nlrp3 inflammasome. Stem Cell Reviews and Reports. https://doi.org/10.1007/s12015-020-10010-z.

74. Ropa, J., Cooper, S., Capitano, M. L., Van’t Hof, W., \& Broxmeyer, H. E. (2020). Human hematopoietic stem, progenitor, and immune cells respond ex vivo to SARS-CoV-2 spike protein. Stem Cell Reviews and Reports. https://doi.org/10.1007/s12015020-10056-z.

75. Ratajczak, M. Z., \& Kucia, M. (2020). SARS-CoV-2 infection and overactivation of Nlrp3 inflammasome as a trigger of cytokine "storm" and risk factor for damage of hematopoietic stem cells. Leukemia, 34(7), 1726-1729. https://doi.org/10.1038/s41375020-0887-9.

76. Sahu, K. K., Siddiqui, A. D., \& Cerny, J. (2020). COVID-19 pandemic and impact on hematopoietic stem cell transplantation. Bone Marrow Transplantation, 55(11), 2193-2195. https://oi. org/10.1038/s41409-020-0913-6.

77. Broxmeyer, H. E., \& Parker, G. C. (2020). Impact of COVID-19 and future emerging viruses on hematopoietic cell transplantation and other cellular therapies. Stem Cells and Development, 29(10), 625-626. https://doi.org/10.1089/scd.2020.0064.

78. Konopleva, M., Mikhail, A., Estrov, Z., Zhao, S., Harris, D., Sanchez-Williams, G., ... Andreeff, M. (1999). Expression and function of leptin receptor isoforms in myeloid leukemia and myelodysplastic syndromes: proliferative and anti-apoptotic activities. Blood, 93(5), 1668-1676. https://doi.org/10.1046/j.13652141.1998.00843.x.

79. Nakao, T., Hino, M., Yamane, T., Nishizawa, Y., Morii, H., \& Tatsumi, N. (1998). Expression of the leptin receptor in human leukaemic blast cells. British Journal of Haematology, 102(3), 740-745. https://doi.org/10.1046/j.1365-2141.1998.00843.x.

80. Ozturk, K., Avcu, F., \& Ural, A. U. (2012). Aberrant expressions of leptin and adiponectin receptor isoforms in chronic myeloid leukemia patients. Cytokine, 57(1), 61-67. https://doi.org/10. 1016/j.cyto.2011.10.004.

81. Gorska, E., Popko, K., \& Wasik, M. (2013). Leptin receptor in childhood acute leukemias. Advances in Experimental Medicine and Biology, 756, 155-161. https://doi.org/10.1007/978-94-0074549-0_20.

82. Wasik, M., Gorska, E., Popko, K., Pawelec, K., Matysiak, M., \& Demkow, U. (2006). The Gln223Arg polymorphism of the leptin receptor gene and peripheral blood/bone marrow leptin level in leukemic children. Journal of Physiology and Pharmacology, 57(Suppl 4), 375-383. https://doi.org/10.1016/j.cyto.2009.07. 006.

83. Mouzaki, A., Panagoulias, I., Dervilli, Z., Zolota, V., Spadidea, P., Rodi, M.,.. . Georgakopoulos, T. (2009). Expression patterns of leptin receptor (OB-R) isoforms and direct in vitro effects of recombinant leptin on OB-R, leptin expression and cytokine secretion by human hematopoietic malignant cells. Cytokine, 48(3), 203-211. https://doi.org/10.1016/j.cyto.2009.07.006.

84. Wex, H., Ponelis, E., Wex, T., Dressendorfer, R., Mittler, U., \& Vorwerk, P. (2002). Plasma leptin and leptin receptor expression in childhood acute lymphoblastic leukemia. International Journal of Hematology, 76(5), 446-452. https://doi.org/10.1007/ BF02982810.

85. Tsiotra, P. C., Pappa, V., Koukourava, A., Economopoulos, T., Tsigos, C., \& Raptis, S. A. (2005). Expression of leptin receptors in mononuclear cells from myelodysplastic syndromes and acute myeloid leukemias. Acta Haematologica, 114(2), 71-77. https:// doi.org/10.1159/000086578.

86. Moschovi, M., Trimis, G., Vounatsou, M., Katsibardi, K., Margeli, A., Damianos, A., \& Papassotiriou, I. (2010). Serial plasma concentrations of adiponectin, leptin, and resistin during therapy in children with acute lymphoblastic leukemia. Journal of Pediatric Hematology/Oncology, 32(1), e8-e13. https://doi.org/ 10.1097/MPH.0b013e3181b8a50c. 
87. Yilmaz, M., Kis, C., Ceylan, N. O., Okan, V., Pehlivan, M., Kucukosmanoglu, E., ... Tarakcioglu, M. (2008). Serum leptin level in acute myeloid leukemia patients. Hematology, 13(1), 21-23. https://doi.org/10.1179/102453308X315771.

88. Bruserud, O., Huang, T. S., Glenjen, N., Gjertsen, B. T., \& Foss, B. (2002). Leptin in human acute myelogenous leukemia: studies of in vivo levels and in vitro effects on native functional leukemia blasts. Haematologica, 87(6), 584-595.https://doi.org/10.1038/ nm. 4252.

89. Lu, Z., Xie, J., Wu, G., Shen, J., Collins, R., Chen, W., \& Zhang, C. C. (2017). Fasting selectively blocks development of acute lymphoblastic leukemia via leptin-receptor upregulation. Nature Medicine, 23(1), 79-90. https://doi.org/10.1038/nm.4252.

90. Kraakman, M. J., Kammoun, H. L., Dragoljevic, D., Al-Sharea, A., Lee, M. K. S., Flynn, M. C.,.. . Murphy, A. J. (2018). Leptindeficient obesity prolongs survival in a murine model of myelodysplastic syndrome. Haematologica, 103(4), 597-606. https://doi.org/10.3324/haematol.2017.181958.

91. Ma, X., Lim, U., Park, Y., Mayne, S. T., Wang, R., Hartge, P., \& Schatzkin, A. (2009). Obesity, lifestyle factors, and risk of myelodysplastic syndromes in a large US cohort. American Journal of Epidemiology, 169(12), 1492-1499. https://doi.org/ 10.1093/aje/kwp074.

92. Murphy, F., Kroll, M. E., Pirie, K., Reeves, G., Green, J., \& Beral, V. (2013). Body size in relation to incidence of subtypes of haematological malignancy in the prospective Million Women Study. British Journal of Cancer, 108(11), 2390-2398. https://doi.org/ 10.1038/bjc.2013.159.

93. Ladas, E. J., Orjuela, M., Stevenson, K., Cole, P. D., Lin, M., Athale, U. H.,.. . Kelly, K. M. (2016). Dietary intake and childhood leukemia: The Diet and Acute Lymphoblastic Leukemia Treatment (DALLT) cohort study. Nutrition (Burbank, Los Angeles County, Calif.), 32(10), 1103-1109 e1101. https://doi. org/10.1016/j.nut.2016.03.014.

94. Decker, M., Martinez-Morentin, L., Wang, G., Lee, Y., Liu, Q., Leslie, J., \& Ding, L. (2017). Leptin-receptor-expressing bone marrow stromal cells are myofibroblasts in primary myelofibrosis. Nature Cell Biology, 19(6), 677-688. https://doi.org/10.1038/ ncb3530.

95. Gupta, M. K., Vethe, H., Softic, S., Rao, T. N., Wagh, V., Shirakawa, J., \& Kulkarni, R. N. (2020). Leptin receptor signaling regulates protein synthesis pathways and neuronal differentiation in pluripotent stem cells. Stem Cell Reports, 15(5), 1067-1079. https://doi.org/10.1016/j.stemcr.2020.10.001.

96. Yang, F., Wu, R., Jiang, Z., Chen, J., Nan, J., Su, S., \& Wang, J. (2018). Leptin increases mitochondrial OPA1 via GSK3-mediated OMA1 ubiquitination to enhance therapeutic effects of mesenchymal stem cell transplantation. Cell Death \& Disease, 9(5), 556. https://doi.org/10.1038/s41419-018-0579-9.
97. Yang, F., Li, B., Yang, Y., Huang, M., Liu, X., Zhang, Y., \& Yang, L. (2019). Leptin enhances glycolysis via OPA1-mediated mitochondrial fusion to promote mesenchymal stem cell survival. International Journal of Molecular Medicine, 44(1), 301-312. https://doi.org/10.3892/ijmm.2019.4189.

98. Sierra-Honigmann, M. R., Nath, A. K., Murakami, C., GarciaCardena, G., Papapetropoulos, A., Sessa, W. C.,... . FloresRiveros, J. R. (1998). Biological action of leptin as an angiogenic factor. Science, 281(5383), 1683-1686. https://doi.org/10.1126/ science.281.5383.1683.

99. Kurtovic, S., Ng, T. T., Gupta, A., Arumugaswami, V., Chaiboonma, K. L., Aminzadeh, M. A., \& Talavera-Adame, D. (2015). Leptin enhances endothelial cell differentiation and angiogenesis in murine embryonic stem cells. Microvascular Research, 97, 65-74. https://doi.org/10.1016/j.mvr.2014.09.004.

100. Crean-Tate, K. K., \& Reizes, O. (2018). Leptin regulation of cancer stem cells in breast and gynecologic cancer. Endocrinology, 159(8), 3069-3080. https://doi.org/10.1210/en.2018-00379.

101. Lipsey, C. C., Harbuzariu, A., Daley-Brown, D., \& GonzalezPerez, R. R. (2016). Oncogenic role of leptin and Notch interleukin-1 leptin crosstalk outcome in cancer. The World Journal of Methodology, 6(1), 43-55. https://doi.org/10.5662/ wjm.v6.i1.43.

102. Zheng, Q., Banaszak, L., Fracci, S., Basali, D., Dunlap, S. M., Hursting, S. D., \& Reizes, O. (2013). Leptin receptor maintains cancer stem-like properties in triple negative breast cancer cells. Endocrine-Related Cancer, 20(6), 797-808. https://doi.org/10. 1530/ERC-13-0329.

103. Thiagarajan, P. S., Zheng, Q., Bhagrath, M., Mulkearns-Hubert, E. E., Myers, M. G., Lathia, J. D., \& Reizes, O. (2017). STAT3 activation by leptin receptor is essential for TNBC stem cell maintenance. Endocrine-Related Cancer, 24(8), 415-426. https://doi. org/10.1530/ERC-16-0349.

104. Wang, T., Fahrmann, J. F., Lee, H., Li, Y. J., Tripathi, S. C., Yue, C., \& Yu, H. (2018). JAK/STAT3-regulated fatty acid betaoxidation is critical for breast cancer stem cell self-renewal and chemoresistance. Cell Metabolism, 27(6), 1357. https://doi.org/10. 1016/j.cmet.2018.04.018.

105. Sasaki, T. (2015). Age-associated weight gain, leptin, and SIRT1: a possible role for hypothalamic SIRT1 in the prevention of weight gain and aging through modulation of leptin sensitivity. Frontiers in Endocrinology (Lausanne), 6, 109. https://doi.org/10. 3389/fendo.2015.00109.

Publisher's Note Springer Nature remains neutral with regard to jurisdictional claims in published maps and institutional affiliations. 